\section{American Journal of Nephrology}

Am J Nephrol 2021;52:559-571

DOI: $10.1159 / 000517852$
Received: March 31, 2021

Accepted: June 14, 2021

Published online: August 13, 2021

\title{
Prognostic and Diagnostic Values of Novel Serum and Urine Biomarkers in Lupus Nephritis: A Systematic Review
}

\author{
Massimo Radin ${ }^{a}$ Paolo Miraglia ${ }^{a, b} \quad$ Alice Barinotti ${ }^{a, b} \quad$ Roberta Fenoglio $^{c}$ \\ Dario Roccatello ${ }^{a, c}$ Savino Sciascia ${ }^{a, c}$ \\ aDepartment of Clinical and Biological Sciences, Center of Research of Immunopathology and Rare Diseases- \\ Coordinating Center of Piemonte and Aosta Valley Network for Rare Diseases, S. Giovanni Bosco Hospital, University \\ of Turin, Turin, Italy; 'b Department of Clinical and Biological Sciences, School of Specialization of Clinical Pathology, \\ University of Turin, Turin, Italy; ' Department of Clinical and Biological Sciences, Nephrology and Dialysis, S. Giovanni \\ Bosco Hospital and University of Turin, Turin, Italy
}

\section{Keywords}

Lupus nephritis - Systemic lupus erythematosus - Biomarker . Urinary $\cdot$ Serological $\cdot$ Systematic review

\begin{abstract}
Introduction: While renal biopsy remains the gold standard for diagnosing lupus nephritis (LN), the prognostic and diagnostic role of non-invasive biomarkers for $L N$ is currently debated. Methods: Available studies published in last 5 years (2015-2020) assessing the diagnostic and prognostic value of urinary and/or serological biomarkers in subjects with LN were analyzed in this systematic review. Results: Eighty-five studies were included (comprehending 13,496 patients with systemic lupus erythematosus [SLE], 8,872 LN, 487 pediatric LN, 3,977 SLE but no LN, 160 pediatric SLE but no LN and 7,679 controls). Most of the studies were cross-sectional (62; $73 \%$ ), while 14 (17\%) were prospective. In sixty studies (71\%), the diagnosis of LN was biopsy-confirmed. Forty-four out of $85(52 \%)$ investigated only serological biomarkers, 29 studies $(34 \%)$ tested their population only with urinary biomarkers, and 12 (14\%) investigated the presence of both. Outcome measures to assess the clinical utility of the analyzed biomarkers were heterogeneous, including up to 21 differ-
\end{abstract}

karger@karger.com

(c) 2021 S. Karger AG, Basel

www.karger.com/ajn

Karger" ent activity scores, with the SLEDAI (in 60\%) being the most used. Despite some heterogeneity, promising results have been shown for biomarkers such as urinary monocyte chemoattractant protein, urinary adiponectin, and urinary vascular cell adhesion protein 1. Discussion/Conclusion: While serum and urine biomarkers have the potential to improve diagnostic and prognostic pathways in patients with $L N$, the vast heterogeneity across studies severely limits their applicability in current clinical practice. With the kidney biopsy still representing the gold standard, future efforts should focus on harmonizing study inclusion criteria and outcomes, particularly in clinical trials, in order to improve comparability and facilitate the implementations of available biomarkers into the daily practice.

(c) 2021 S. Karger AG, Basel

\section{Significance Statement}

While kidney biopsy is crucial for lupus nephritis (LN) diagnosis, the identification of one or more non-invasive biomarkers (serological or urinary) is highly needed. Potentially, the study of novel biomarkers may be useful to provide further information on LN: mainly the degree of 
disease activity, early recognition of renal flares, guide treatment selection, and responsiveness to the therapy. In this systematic review, we analyzed 85 studies comprehending 13,496 patients with systemic lupus erythematosus (SLE) and 8,872 with LN. Despite some heterogeneity, promising results have been shown for biomarkers such as urinary monocyte chemoattractant protein, urinary adiponectin, and urinary vascular cell adhesion protein 1 . This could pave the way to a new era of precision medicine in the treatment and management of LN, ultimately improving the prognosis of patients.

\section{Introduction}

Systemic lupus erythematosus (SLE) is a chronic autoimmune disease characterized by the production of autoantibodies directed against nuclear components (e.g., anti-dsDNA, anti-SSA/Ro, anti-SSB/La, anti-Sm, and antiRNP) and soluble mediators. The binding of these autoantibodies to their antigens generates immune complexes that can be deposited and can damage different organs, causing a wide variety of clinical manifestations [1]. The kidney is one of the main organs affected (50$80 \%$ of SLE patients), and its involvement, LN, represents a major cause of morbidity and mortality in SLE patients $[2,3]$. Several events, such as complement activation, aberrant apoptosis, and genetic predisposition, concur in the loss of self-tolerance that leads to these autoantibody production and immune complex deposition, thus supporting LN development and progression [4-6].

Despite the presence of guidelines regarding diagnosis and management, standard-of-care immunosuppression can fail and LN can progress to end-stage renal disease within 5 years after the onset [7].

Renal biopsy, an invasive procedure, is the gold standard for the diagnosis of LN and to determine the degree of glomerular, tubular, vascular, and interstitial involvement [8]. Similarly, its utility in guiding the choice of one immunosuppressive therapy over another is still limited [9]. This is made more difficult by the high degree of heterogeneity existing among patients with SLE. Alterations in specific pathways, and in this case in immune pathways, can contribute more than others to the development and progression of the disease in a certain patient. The use of biomarkers seems to be a valuable approach to overcome these issues.

Biomarkers represent biological characteristics that can be evaluated as indicators of physiological processes, pathological processes, or responsiveness to therapy [10]. The conventional prognostic and diagnostic biomarkers for LN include primarily proteinuria [11], as well as elevated levels of serum creatinine, hematuria, urinary cellular casts, antidouble-stranded DNA, and complement levels.

Recently, we have experienced a growing body of evidence investigating the potential prognostic and diagnostic role of genetic polymorphisms and protein detectable in serum and/or urine samples to guide the management of LN [12]. Among the latter ones, there are proteins involved in the immune response, such as cytokines, chemokines, and complement factors and other proteins highly expressed by the glomerular and tubular injured cells, such as adhesion molecules and growth factors $[13$, 14].

Thus, while a kidney biopsy is crucial for LN diagnosis, the identification of one or more biomarkers would be useful to provide further information on the degree of disease activity, early recognition of renal flares, guide treatment selection, and responsiveness to the therapy. Ideally, the future goal would be the development of specific approaches for each patient as we are experiencing in the field of oncology in the era of precision medicine. The aim of this systematic review was to study the clinical utility of serological and urinary biomarkers that have been investigated in the field of LN in the last 5 years.

\section{Methods}

A detailed literature search has been developed a priori to identify articles that reported findings from clinical and laboratory studies that analyzed the prognostic role, diagnostic role, and the role in the evaluation of the therapy response of biomarkers (either urinary of serological) in patients with SLE and LN. Keywords and subject terms included ("lupus nephritis" [MeSH Terms] OR ("lupus" [All Fields] AND “nephritis" [All Fields]) OR "lupus nephritis" [All Fields]) AND ("biomarkers" [MeSH Terms] OR "biomarkers" [All Fields]) AND (“April 25, 2015” [PDat]: “April 22, 2020" [PDat]). The search strategy was applied to Ovid MEDLINE, In-Process and Other Non-Indexed Citation and Ovid MEDLINE for the last 5 years (from July 2015 to 2020). Abstracts from EULAR and ACR/ARHP Annual Meetings (20152020) were screened and included in the analysis when meeting the inclusion criteria and not replicating studies published elsewhere.

Inclusion criteria of the studies were as follows:

1. Studies that included at least 50 SLE patients of which at least 25 were LN sufferers.

2. Urinary and/or serological testing of novel* biomarkers.

Studies that met the inclusion criteria were systematically analyzed by 2 independent reviewers (M.R. and P.M.). Disagreements were resolved by consensus; if consensus could not be achieved, a third party (S.S.) would provide an assessment of eligibility. As the data on eligibility were dichotomous (eligible: yes/no), agreement 


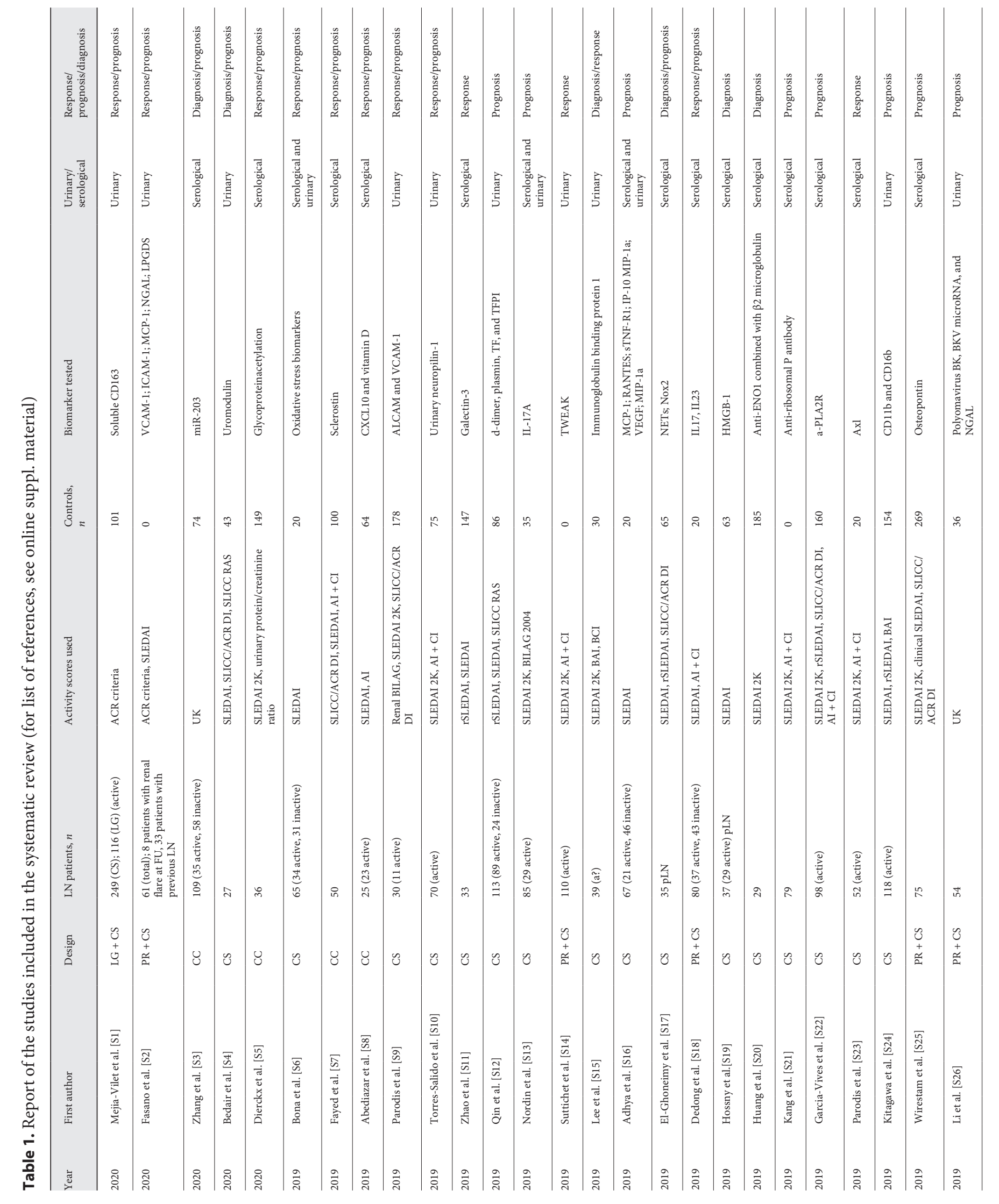




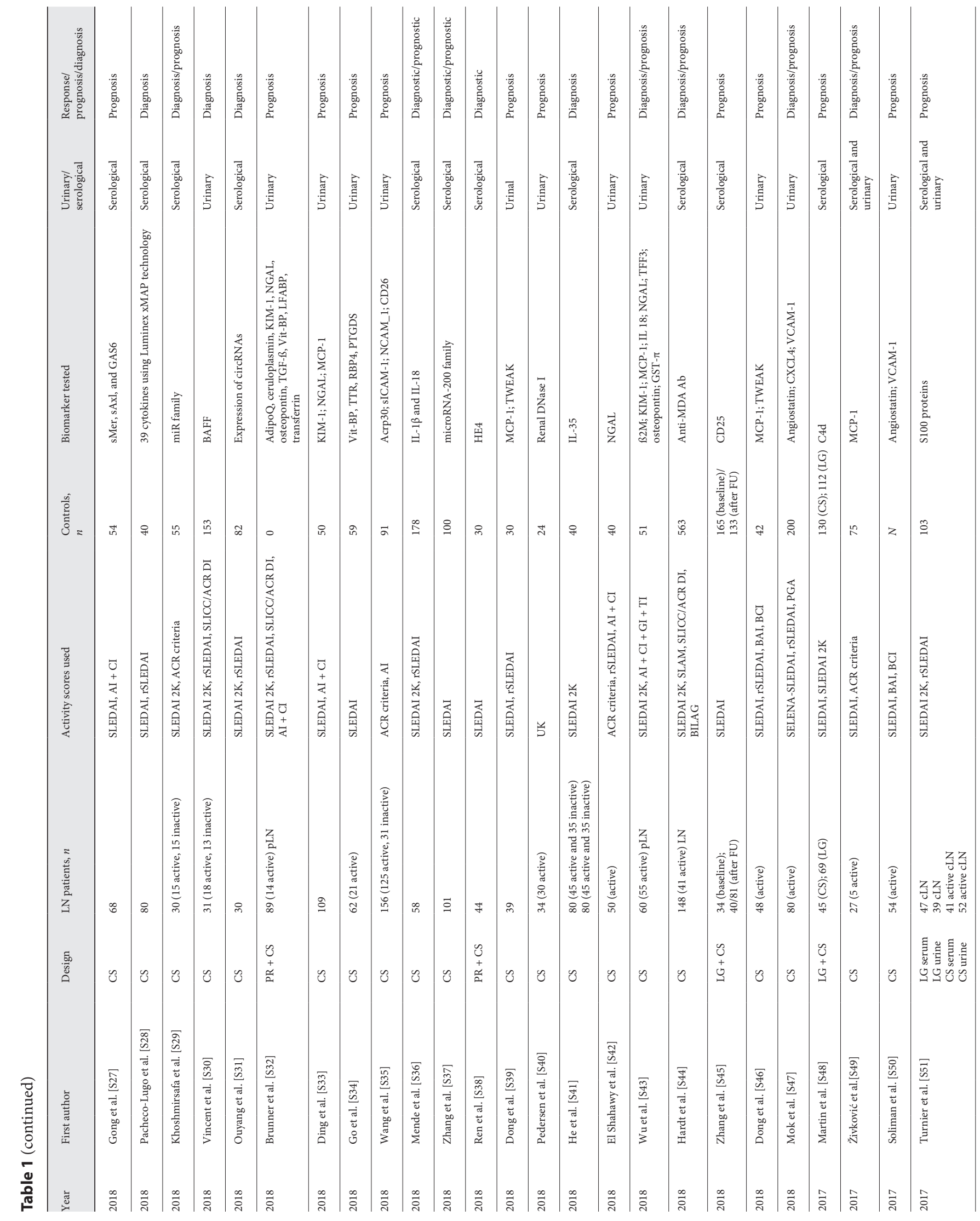



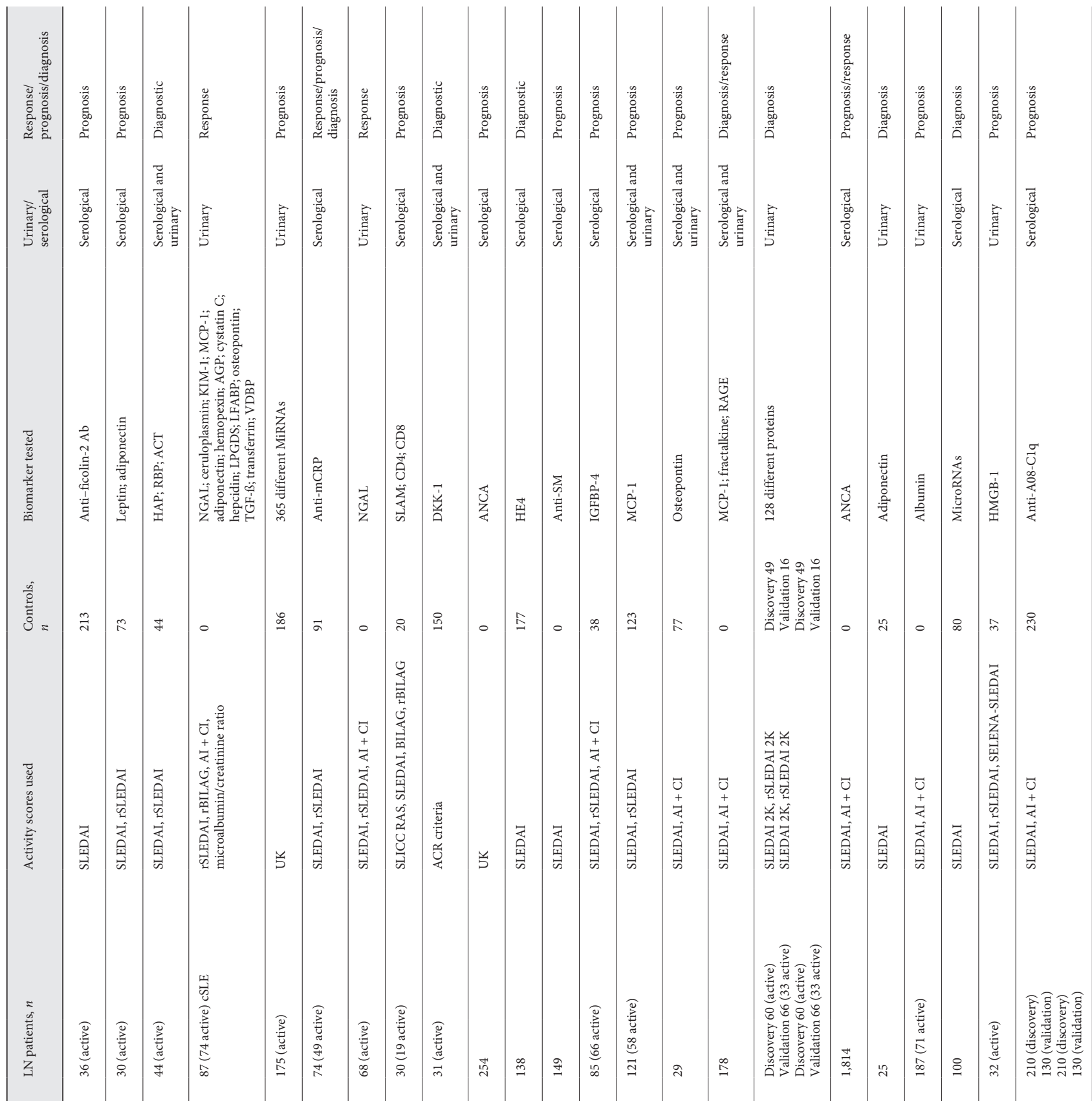

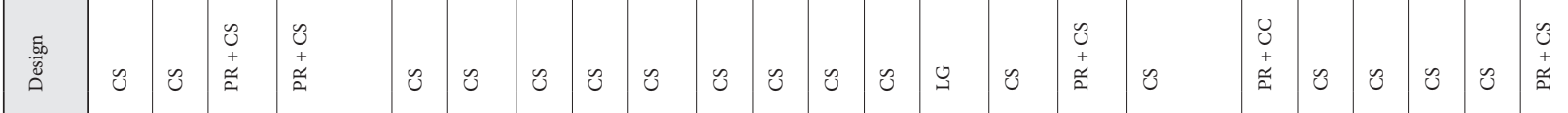

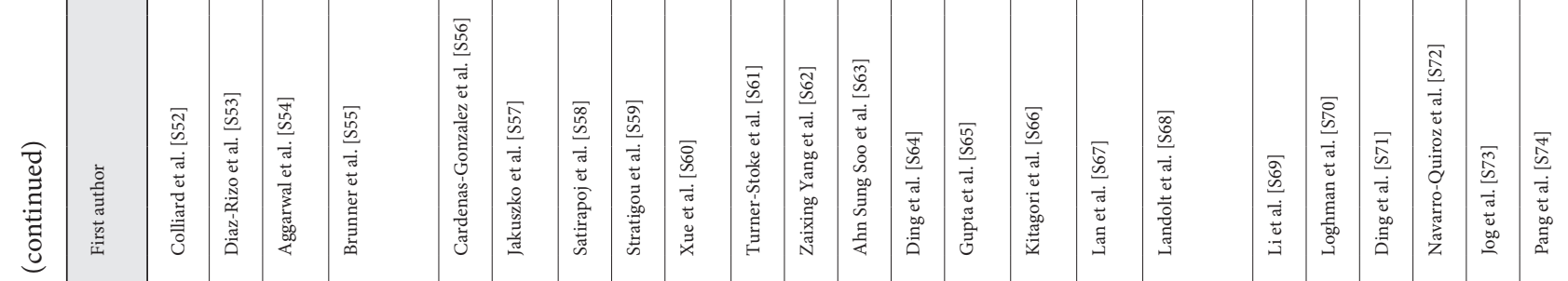

息| 


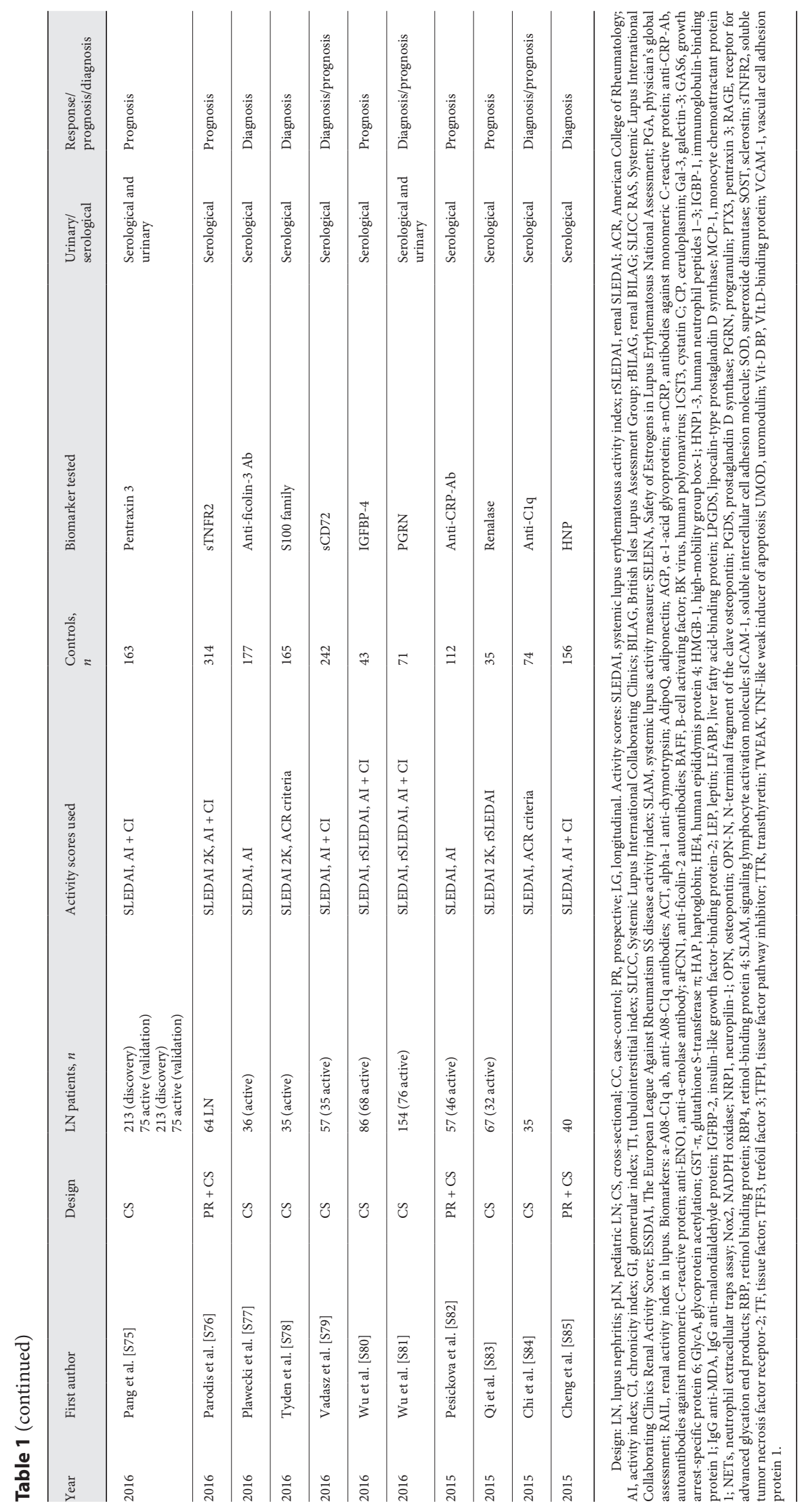


Table 2. Summary of the activity scores used in the studies included in the systematic review (for list of references, see online suppl. material)

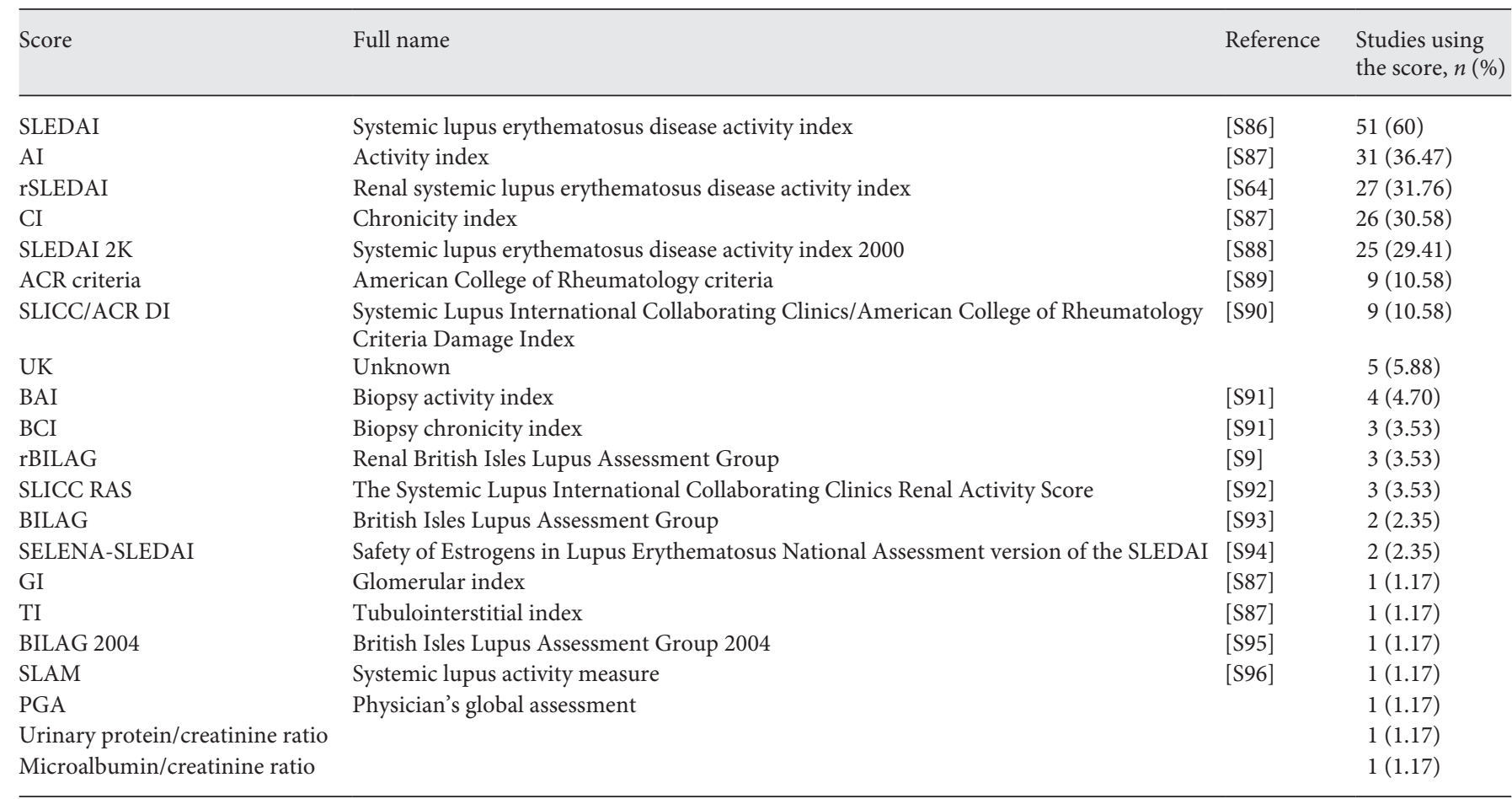

at both the title and abstract review and the full article review stages was determined by calculation of Cohen's kappa coefficient $(k=0.9)$. The literature search strategy is shown in online suppl. Fig. 1S (see www.karger.com/doi/10.1159/000517852 for all online suppl. material).

The present study has been performed according to PRISMA guidelines [15]. Studies that tested only the presence of serological or urinary biomarkers that are recommended in the current guidelines $[16,17]$ for LN were excluded.

\section{Results}

Data from 13,496 SLE patients included in 85 retrieved studies were analyzed (references of the included studies are available in the online suppl. material as "online suppl. references"). The retrieved studies had different designs and varying sample sizes. Table 1 displays the main characteristics of the studies included in the analysis, the enrolled populations, study design, activity scores used, studied biomarker, and the role of the latter.

When considering the populations included in the studies that met the inclusion criteria of the systematic review, a total of 8,872 patients with SLE and LN (1,573 patients with active LN, 2,906 with inactive LN, and
4,393 cases in which the activity of $\mathrm{LN}$ was not addressed in the study) and a total of 487 pediatric patients with SLE and LN were analyzed. As controls, a total of 3,977 patients with SLE but no LN were included, as well as 160 pediatric patients with SLE and without renal involvement. Totally, the studies included 7,679 controls, comprehending 2,457 healthy donors, 268 patients with glomerulopathies but not LN, 154 patients with autoimmune conditions but not SLE, and 34 patients with diabetic nephropathy.

When focusing on study design, 62 studies used a cross-sectional approach, 14 studies were prospective, 4 studies were case-control, 4 studies were longitudinal and cross-sectional, and 1 study was longitudinal. Considering the definition of LN, renal biopsy was used as a gold standard in 60 studies (71\%), while in 9 studies (11\%), histological confirmation was not performed, in 9 studies (11\%), the information on renal biopsy could not be retrieved [18-25], and in 7 studies (8\%), renal biopsy was performed only in a subgroup of the investigated SLE patients included in the analysis.

When focusing on the tool used to assess activity of LN, 21 different activity scores have been used in the analyzed studies, as summarized in Table 2. In particular, 
Table 3. Combinations of activity scores used in the studies included in the systematic review.

\begin{tabular}{llcl}
\hline Scores used, $n$ & Type of scores primarily used & Studies, $n(\%)$ & \\
\hline 1 & SLEDAI & $12(75)$ & $16(18.82)$ \\
& ACR criteria & $2(12.5)$ & $24(28.23)$ \\
\hline SLEDAI 2K & $2(12.5)$ & $7(29.17)$ \\
& SLEDAI + rSLEDAI & $5(20.83)$ & $27(31.77)$ \\
& SLEDAI 2K + rSLEDAI & $3(12.5)$ & \\
\hline 3 & SLEDAI + AI & $3(12.5)$ & \\
& SLEDAI + ACR criteria & $6(25)$ & \\
\hline 4 & Other combinations & $11(40.74)$ & $5(18.52)$ \\
\hline SLEDAI + AI + CI & $11(40.74)$ & \\
\hline SLEDAI 2K + AI + CI & Other combinations & $9(10.6)$ & \\
\hline
\end{tabular}

SLEDAI, systemic lupus erythematosus activity index; rSLEDAI, renal SLEDAI; SLEDAI 2K, SLEDAI 2000; ACR, American College of Rheumatology; AI, activity index; CI, chronicity index.

the most used resulted to be systemic lupus erythematosus activity index - SLEDAI (51 studies out of $85=60 \%$ ), activity index - AI ( 31 out of $85=36.47 \%)$, renal Systemic Lupus Erythematosus Activity Index - rSLEDAI (27 out of $85=31.76 \%)$, chronicity index - CI ( 26 out of $85=$ $30.58 \%$ ), and Systemic Lupus Erythematosus Activity Index 2000 - SLEDAI 2 K ( 25 out of $85=29.41 \%)$. Moreover, the majority of the studies ( 69 out of $85=81.18 \%$ ) combined the use of $>1$ score. More details about their combination are shown in Table 3.

When analyzing the novel biomarkers, 44 out of 85 (52\%) investigated only serological biomarkers, while 29 studies (34\%) tested their population only with urinary biomarkers. Moreover, 12 studies (14\%) investigated the presence of novel biomarkers in both urine and blood. Interestingly, 5 studies (6\%) tested the investigated biomarkers (serological or urinary) also on the renal biopsy specimens. Last, regarding the clinical utility of the biomarker, 59 studies tested the biomarkers to evaluate the activity and long-term prognosis/renal outcome of LN, 36 studies tested the biomarkers during the diagnostic process, and 14 studies tested the biomarkers in correlation with response to therapy.

Table 4 resumes the serological and urinary biomarkers investigated in the studies included in the systematic review. The most researched biomarkers are discussed separately.

\section{Monocyte Chemoattractant Protein-1 (MCP-1)}

MCP-1 has been used as biomarker in $18(21 \%)$ of the included studies. In particular, in 13 studies (15\%), it has been analyzed in urine samples, and in 5 studies (6\%), it has been analyzed in serum samples. MCP-1 belongs to the $\mathrm{C}-\mathrm{C}$ chemokine family. Even if it is produced by many cell types, it is mainly expressed by activated monocytes/macrophages, $\mathrm{T}$ cells, and natural killer cells and takes part in leukocyte infiltration to the kidney [26]. This and the observation that its antagonist can ameliorate the induction and progression of $\mathrm{LN}$ in transgenic mouse model [27], make it one of the most used biomarkers when considering LN. As shown in online suppl. Table 1S, while 7 out of 13 studies found a potential diagnostic or prognostic role for this marker in the context of LN, still a certain level of disagreement exists. In fact, 5 studies out of 13 reported an association between the presence of the increased level of this biomarkers and LN. When looking at its prognostic role using renal disease activity as an outcome, 3 studies showed a negative correlation with MCP-1 and 8 demonstrated a positive correlation (2 studies did not investigate upon this aspect). Further prospective analyses are needed to the additional value, ideally as both diagnostic and prognostic perspectives. 


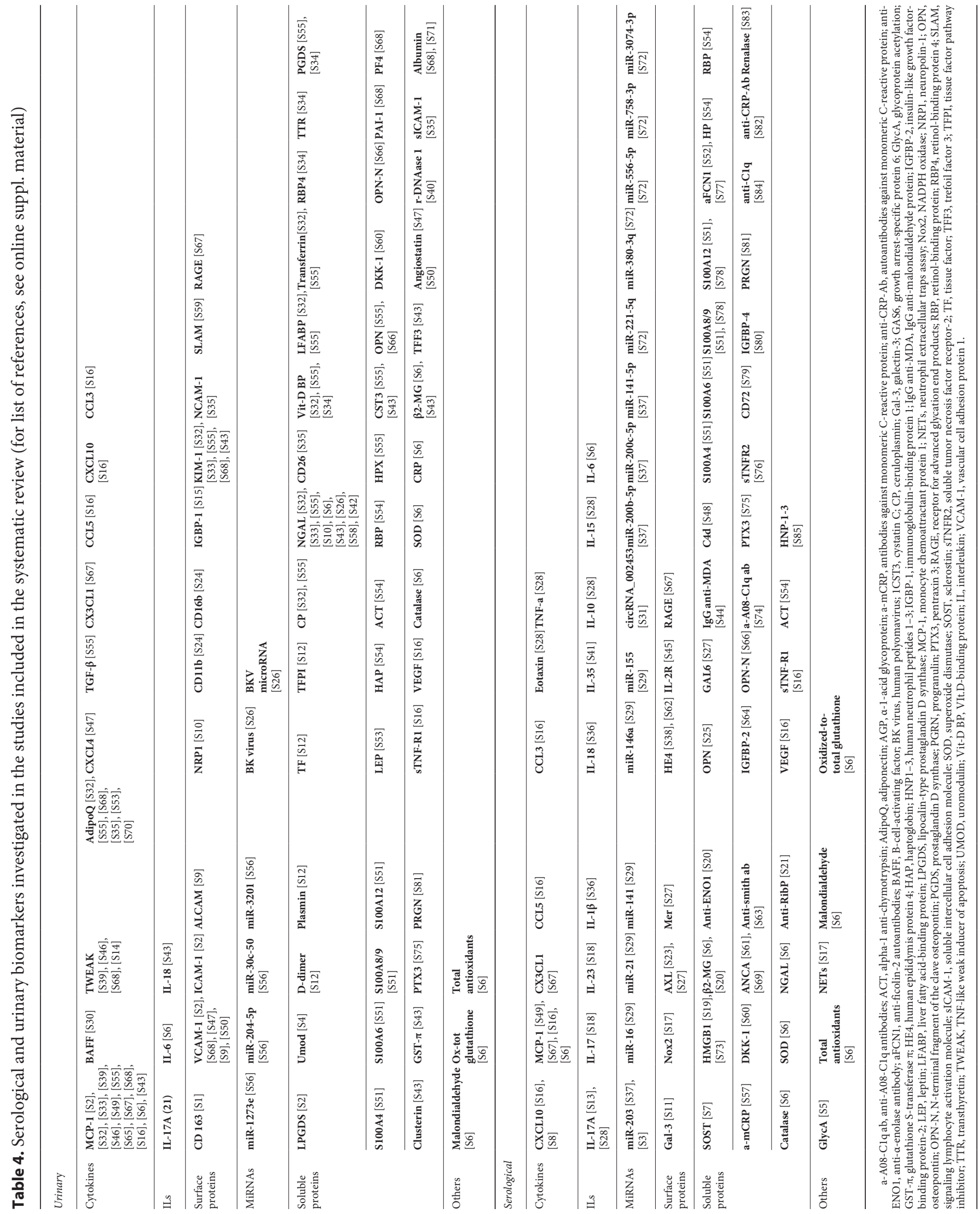


Urinary Vascular Cell Adhesion Protein 1 (VCAM-1)

VCAM-1 has been used as a urinary biomarker in 5 of the analyzed studies (6\%). It represents an adhesion molecule that is involved in the recruitment of inflammatory cells by its interaction with infiltrating leukocytes. Its soluble levels appear elevated in autoimmune disease, such as SLE and rheumatoid arthritis. Moreover, when considering SLE patients, it has been demonstrated an even more pronounced increase of urinary VCAM-1 in patients with LN [28]. Details are shown in Table 3. When compared to other biomarkers, albeit limited in numbers, available studies seem to show a good level of consistency in supporting its role as a potential marker in $\mathrm{LN}$.

\section{Urinary Adiponectin (AdipoQ)}

AdipoQ has been used as a urinary biomarker in 5 of the analyzed studies (6\%). It is a small adipocytokine presenting both anti- and pro-inflammatory functions. When considering patients suffering from LN, and in particular active LN, adiponectin results to be highly expressed in glomeruli and renal tubules and shows an increase of its urinary levels, not found in SLE patients without renal involvement $[29,30]$.

\section{Urinary Kidney Injury Molecule-1 (Kim-1)}

Kim-1 has been used as a urinary biomarker in 5 of the included studies $(6 \%)$. It is a transmembrane protein of proximal tubule cells whose expression is strongly upregulated during tubule damage. Recent studies highlighted an increase of Kim-1 urinary levels in the presence of active $\mathrm{LN}$ and then a new decline during the remission phase [31].

\section{Urinary TNF-Like Weak Inducer of Apoptosis}

(TWEAK)

Urinary levels of TWEAK have been analyzed in 5 of the included studies $(6 \%)$. This protein belongs to the TNF receptor superfamily. It plays a role in chronic inflammation and, in particular, shows a close correlation with renal inflammation. Also in this case, its urinary levels seem to be markedly elevated during the active phase of LN and to undergo a decrease during the remission phase [26].

\section{Urinary Neutrophil Gelatinase-Associated Lipocalin}

(NGAL)

NGAL has been used as a urinary biomarker in 8 of the analyzed studies $(9.5 \%)$. It is a soluble protein secreted during conditions of stress and inflammation by leukocytes and tubular epithelial cells. Several studies high- lighted how NGAL can help predicting acute kidney injury and that high urinary levels can be detected among SLE patients suffering from LN [21]. However, 3 out of 8 retrieved studies analyzing the clinical accuracy of NGAL did not report any conclusive results.

\section{Discussion/Conclusion}

To date, the gold standard for diagnosing LN remains renal biopsy. The histological findings not only are fundamental for diagnosis but also guide treatment options [32]. From this perspective, some considerations are worth noting. First, kidney biopsy is overall a safe procedure if performed in experienced centers, even in patients with autoimmune diseases [8]. Second, the use of protocolized repeated biopsy is currently debated, and an international study is currently ongoing [33]. Available evidence is suggesting that the per-protocol repeating of kidney biopsy might provide the treating physicians with critical information to further guide the management (e.g., by assessing the degree of glomerular sclerosis and/ or histological features of response to treatment $[9,34$, 35]. To date, the question if kidney biopsy should be repeated per-protocol or performed "on demand" in case of clinical changes remains to date open. The challenge for the future is to implement our ability to management LN by using noninvasive biomarkers $[36,37]$. Tests able to improve our abilities to accurately predict future renal flares are highly needed. Similarly, biomarkers allowing the identification of ongoing LN earlier than currently available tools (e.g., proteinuria, hematuria, and increased serum creatinine levels) have the potential to improve our diagnosis accuracy [38].

Our study systematically reviewed the current literature on novel potential biomarker for diagnosing $\mathrm{LN}$ and monitoring renal disease activity. On the one hand, considering the stringent inclusion criteria of the included studies, onecould point out that the evidence on potential novel biomarkers of interest in LN is exponentially growing, making it a dynamic research topic. On the other hand, the analysis of the included studies points out various aspects that should still be improved when investigating biomarkers in LN. First, in about a third of the studies, LN was not confirmed by kidney biopsy. Second, methodologically, there was a vast heterogeneity when evaluating together the used tools to assess the activity of the LN. Third, we observed a strong intra- and inter-assay variance in terms of laboratory testing across the analyzed studies. 
One should consider that despite the mentioned level of heterogeneity, some promising results have been shown for some biomarkers such as urinary MCP-1, urinary AdipoQ, and urinary VCAM-1. Other promising biomarkers, despite not being the most frequently investigated in the included studies, are worth mentioning: the activated leukocyte cell adhesion molecule (ALCAM), the soluble marker CD163, and the B lymphocyte stimulator (BLyS). Recent studies indeed highlighted ALCAM and CD163 correlation with renal biopsy outcomes and their effectiveness in distinguishing among active and inactive LN [39-42]. When focusing on BLyS, even if it does not seem to correlate with the activity or quiescence of the disease, it resulted to be highly expressed in LN patients [43-46]. BLyS is a B-cell survival factor showing a pivotal role in $\mathrm{B}$ cell maturation and activation, whose expression is closely correlated with autoimmunity. These observations are in line with the recent approval of the monoclonal antibody targeting BLyS for the management of SLE. All in all, a growing body of evidence is exploring the role of ALCAM, CD136, and BLyS/BAFF to guide the different phases of the management of LN. Available evidence is still limited to a small number of studies; however, future research is highly expected to confirm the promising preliminary results. That is why the monoclonal antibody targeting BLyS (belimumab) has been recently approved for the management of SLE [47].

The role of cytokines in the pathogenesis of SLE has been largely investigated. Among others, interleukin (IL)-6, IL-17, IL-12, and transforming growth factor $\beta$ (TGF- $\beta$ ) are the cytokines of greater interest when considering LN. A growing body of evidence is suggesting their potential role as prognostic and/or diagnostic biomarkers [48-51]. For instance, IL-6 and IL-17 levels have been observed to correlate with the disease activity and remission $[48,50]$. However, to date, these results still require validation in large prospective cohorts. Besides, some technical aspects, including inter-assay variability, need to be addressed before considering this candidate as part of the clinical routine investigations. Nevertheless, the availability of therapeutic options directly targeting different cytokines is likely to further promote this area of research.

Nevertheless, while repetitive sampling to unmask the evolution of a LN and/or assess treatment response is only possible with noninvasively tools (e.g., urine-based), the "liquid biopsy" is still the Holy Grail for "lupologist" community. In fact, some pilot results have shown how urine proteomics approaches can identify an inflamma- tory signature in the urine of patients with LN implicating monocyte and TNF- $\alpha$ pathways. These signatures are associated with proliferative disease, worse proteinuria, and nonresponse to treatment [52]. If confirmed, these results may pave the way for a more personalized approach to LN treatment.

This study suffers some limitations, mainly due to the heterogeneity of the studies included in the analysis. Importantly, all of the included studies were observational studies, subject to the biases inherent in such study designs, and the reproducibility of their results might suffer from it. When considering the analyzed data, the heterogeneity in the data of the studies comprehended inclusion criteria, biomarkers and assay heterogeneity, cutoff value definition, clinical details of the patients and diagnosis of LN, control groups, and definition of renal activity disease. Moreover, it was not possible to perform a meta-analysis of the studies (even when considering the biomarkers investigated in different studies due to lack of details limiting comparability).

Autoantibodies, as well as other protein biomarkers, cytokines, and chemokines, clearly play a pivotal role in SLE pathogenesis and could potentially be used as biomarkers. While the value of identifying a biomarker for the diagnosis on LN and disease monitoring would represent a cornerstone in SLE management, this could also potentially represent the basis for future therapeutic approaches.

In order to further proceed in this very promising field of research, clinical trial design in LN should be further improved and homogenized in order to be able to compare outcomes among studies and improve result interpretation. To date, kidney biopsy still represents the most accurate tool to confirm LN diagnosis and to obtain prognostic data through a proper histological evaluation.

\section{Statement of Ethics}

Due to the nature of the article (systematic review) the study did not need the approval by the local ethical committee.

\section{Conflict of Interest Statement}

The authors have no conflicts of interest to disclose. 


\section{Funding Sources}

The authors did not receive any funding.

\section{Author Contributions}

M.R., P.M., and A.B. searched the literature, assisted with the organization of the manuscript, interpreted and collected data, and wrote and edited the review. R.F., D.R., and S.S. interpreted and collected data, helped to design the figures and panel, and wrote and edited the review.

\section{References}

1 Tsokos GC. Mechanisms of disease: systemic lupus erythematosus. N Engl J Med. 2011; 365(22):2110-21121.

2 Almaani S, Meara A, Rovin BH. Update on lupus nephritis. Clin J Am Soc Nephrol. 2016; 12(5):825.

3 Rovin BH, Parikh SV. Lupus nephritis: the evolving role of novel therapeutics. Am J Kidney Dis. 2014;63(4):677-90.

4 Yu F, Haas M, Glassock R, Zhao MH. Redefining lupus nephritis: clinical implications of pathophysiologic subtypes. Nat Rev Nephrol. 2017;13(8):483-95.

5 Anders HJ, Rovin B. A pathophysiologybased approach to the diagnosis and treatment of lupus nephritis. Kidney Int. 2016; 90(3):493-501.

6 Lech M, Anders HJ. The pathogenesis of lupus nephritis. J Am Soc Nephrol. 2013;24(9): 1357-66.

7 Tektonidou MG, Dasgupta A, Ward MM. Risk of end-stage renal disease in patients with lupus nephritis, 1971-2015: a systematic review and bayesian meta-analysis. Arthritis Rheumatol. 2016;68(6):1432-41.

8 Roccatello D, Sciascia S, Rossi D, Naretto C, Bazzan M, Solfietti L, et al. Outpatient percutaneous native renal biopsy: safety profile in a large monocentric cohort. BMJ Open. 2017; 7(6):e015243

9 Restrepo-Escobar M, Granda-Carvajal PA, Jaimes F. Systematic review of the literature on reproducibility of the interpretation of renal biopsy in lupus nephritis. Lupus. 2017; 26(14):1502-12.

10 Davidson A, Aranow C, Mackay M. Lupus nephritis: challenges and progress. Curr Opin Rheumatol. 2019;31(6):682-8.

11 Dall'Era M, Cisternas MG, Smilek DE, Straub L, Houssiau FA, Cervera R, et al. Predictors of long-term renal outcome in lupus nephritis trials: lessons learned from the euro-lupus nephritis cohort. Arthritis Rheumatol. 2015; 67(5):1305-13.

12 Aragón CC, Tafúr RA, Suárez-Avellaneda A, Martínez MT, Salas A, Tobón GJ. Urinary biomarkers in lupus nephritis. J Transl Autoimmun. 2020;3:100042.

13 Qi S, Chen Q, Xu D, Xie N, Dai Y. Clinical application of protein biomarkers in lupus erythematosus and lupus nephritis. Lupus. 2018 27(10):1582-90.

14 Caster DJ, Powell DW. Utilization of biomarkers in lupus nephritis. Adv Chronic Kidney Dis. 2019;26(5):351-9.
15 http: //prisma-statement.org/documents/ PRISMA\%202009\%20checklist.pdf.

16 Fanouriakis A, Kostopoulou M, Cheema K, Anders HJ, Aringer M, Bajema I, et al. 2019 update of the joint european league against rheumatism and European renal associationeuropean dialysis and transplant association (EULAR/ERA-EDTA) recommendations for the management of lupus nephritis. Ann Rheum Dis. 2020;79(6):S713-S723.

17 Fanouriakis A, Kostopoulou M, Alunno A, Aringer M, Bajema I, Boletis JN, et al. 2019 update of the EULAR recommendations for the management of systemic lupus erythematosus. Ann Rheum Dis. 2019;78(6):736-45.

18 Nordin F, Shaharir SS, Abdul Wahab A, Mustafar R, Abdul Gafor AH, Mohamed Said MS, et al. Serum and urine interleukin-17A levels as biomarkers of disease activity in systemic lupus erythematosus. Int J Rheum Dis. 2019; 22(8):1419-26.

19 Torres-Salido MT, Sanchis M, Solé C, Moliné T, Vidal M, Vidal X, et al. Urinary neuropilin-1: a predictive biomarker for renal outcome in lupus nephritis. Int J Mol Sci. 2019; 20(18):1-19.

20 Dong XW, Zheng ZH, Ding J, Luo X, Li ZQ, $\mathrm{Li}$ Y, et al. Combined detection of UMCP-1 and UTWEAK for rapid discrimination of severe lupus nephritis. Lupus. 2018;27(6):97181.

21 Satirapoj B, Kitiyakara C, Leelahavanichkul A, Avihingsanon Y, Supasyndh O. Urine neutrophil gelatinase-associated lipocalin to predict renal response after induction therapy in active lupus nephritis. BMC Nephrol. 2017; 18(1):263-8

22 Brunner HI, Bennett MR, Gulati G, Abulaban K, Klein-Gitelman MS, Ardoin SP, et al. Urine biomarkers to predict response to lupus nephritis therapy in children and young adults. J Rheumatol. 2017;44(8):1239-48.

23 Martin M, Smolag KI, Björk A, Gullstrand B, Okroj M, Leffler J, et al. Plasma C4d as marker for lupus nephritis in systemic lupus erythematosus. Arthritis Res Ther. 2017;19(1): 1-9.

24 Tydén H, Lood C, Gullstrand B, Jönsen A, Ivars F, Leanderson T, et al. Pro-inflammatory $\mathrm{S} 100$ proteins are associated with glomerulonephritis and anti-dsDNA antibodies in systemic lupus erythematosus. Lupus. 2017; 26(2):139-49

25 Plawecki M, Lheritier E, Clavarino G, JourdeChiche N, Ouili S, Paul S, et al. Association between the presence of autoantibodies targeting Ficolin-3 and active nephritis in patients with systemic lupus erythematosus. PLoS One. 2016;11(9):1-15.

26 Dong X, Zheng Z, Luo X, Ding J, Li Y, Li Z, et al. Combined utilization of untimed single urine of MCP-1 and TWEAK as a potential indicator for proteinuria in lupus nephritis: a case-control study. Med. 2018;97(16):1-7.

27 Živković V, CvetkovićT, Mitić B, Stamenković B, Stojanović S, Radovanović-Dinić B, et al. Monocyte chemoattractant protein-1 as a marker of systemic lupus erythematosus: an observational study. Rheumatol Int. 2018; 38(6):1003-8.

28 Gasparin AA, de Andrade NPB, Hax V, Palominos PE, Siebert M, Marx R, et al. Urinary soluble VCAM-1 is a useful biomarker of disease activity and treatment response in lupus nephritis. BMC Rheumatol. 2020;4(1):67.

29 Brunner HI, Gulati G, Klein-Gitelman MS, Rouster-Stevens KA, Tucker L, Ardoin SP, et al. Urine biomarkers of chronic kidney damage and renal functional decline in childhoodonset systemic lupus erythematosus. Pediatr Nephrol. 2019;34(1):117-28.

30 Loghman M, Haghighi A, Broumand B, Ataipour Y, Tohidi M, Marzbani C, et al. Association between urinary adiponectin level and renal involvement in systemic lupus erythematous. Int J Rheum Dis. 2016;19(7):67884.

31 Ding Y, Nie LM, Pang Y, Wu WJ, Tan Y, Yu $\mathrm{F}$, et al. Composite urinary biomarkers to predict pathological tubulointerstitial lesions in lupus nephritis. Lupus. 2018;27(11):1778-89.

32 Bajema IM, Wilhelmus S, Alpers CE, Bruijn JA, Colvin RB, Cook HT, et al. Revision of the international society of nephrology/renal pathology society classification for lupus nephritis: clarification of definitions, and modified national institutes of health activity and chronicity indices. Kidney Int. 2018;93(4): 789-96.

33 ClinicalTrials.gov [Internet]. Parodis (MD) National Library of Medicine (US). (2020 Jun 29 - ). Per-protocol Repeat Kidney Biopsy in Incident Cases of Lupus Nephritis (REBIOLUP).Identifier NCT04449991. https://clinicaltrials.gov/ct2/show/record/ NCT04449991.

34 Giannico G, Fogo AB. Lupus nephritis: is the kidney biopsy currently necessary in the management of lupus nephritis? Clin J Am Soc Nephrol. 2013;8(1):138-45. 
35 Parodis I, Adamichou C, Aydin S, Gomez A, Demoulin N, Weinmann-Menke J, et al. Perprotocol repeat kidney biopsy portends relapse and long-term outcome in incident cases of proliferative lupus nephritis. Revmatol. 2020;59(11):3424-34.

36 Anders HJ, Saxena R, Zhao M, Parodis I, Salmon JE, Mohan C. Lupus nephritis. Nat Rev Dis Prim. 2020;6(1):7.

37 Gatto M, Saccon F, Zen M, Iaccarino L, Doria A. Preclinical and early systemic lupus erythematosus. Best Pract Res Clin Rheumatol. 2019;33(4):101422.

38 Giacomelli R, Afeltra A, Alunno A, BartoloniBocci E, Berardicurti O, Bombardieri M, et al. Guidelines for biomarkers in autoimmune rheumatic diseases: evidence based analysis. Autoimmun Rev. 2019;18(1):93-106.

39 Stanley S, Vanarsa K, Soliman S, Habazi D, Pedroza C, Gidley G, et al. Comprehensive aptamer-based screening identifies a spectrum of urinary biomarkers of lupus nephritis across ethnicities. Nat Commun. 2020 May 4; 11(1):2197.

40 Parodis I, Gokaraju S, Zickert A, Vanarsa K, Zhang T, Habazi D, et al. ALCAM and VCAM-1 as urine biomarkers of activity and long-term renal outcome in systemic lupus erythematosus. Rheumatology. 2020 Sep 1; 59(9):2237.

41 Ding H, Lin C, Cai J, Guo Q, Dai M, Mohan $\mathrm{C}$, et al. Urinary activated leukocyte cell adhesion molecule as a novel biomarker of lupus nephritis histology. Arthritis Res. Ther. 2020; 22:122.
42 Mejia-Vilet JM, Zhang XL, Cruz C, CanoVerduzco ML, Shapiro JP, Nagaraja HN, et al. Urinary soluble CD163: a novel noninvasive biomarker of activity for lupus nephritis. J Am Soc Nephrol. 2020;31(6):1335-47.

43 Vincent FB, Northcott M, Hoi A, Mackay F, Morand EF. Association of serum B cell activating factor from the tumour necrosis factor family (BAFF) and a proliferation-inducing ligand (APRIL) with central nervous system and renal disease in systemic lupus erythematosus. Lupus. 2013;22(9):873-84.

44 Neusser MA, Lindenmeyer MT, Edenhofer I, Gaiser S, Kretzler M, Regele H, et al. Intrarenal production of B-cell survival factors in human lupus nephritis. Mod Pathol. 2011;24(1): 98-107.

45 Parodis I, Zickert A, Sundelin B, Axelsson M, Gerhardsson J, Svenungsson E, et al. Evaluation of B lymphocyte stimulator and a proliferation inducing ligand as candidate biomarkers in lupus nephritis based on clinical and histopathological outcome following induction therapy. Lupus Sci Med. 2015;2:61.

46 Ritterhouse LL, Crowe SR, Niewold TB, Merrill JT, Roberts VC, Dedeke AB, et al. B lymphocyte stimulator levels in systemic lupus erythematosus: higher circulating levels in African American patients and increased production after influenza vaccination in patients with low baseline levels. Arthritis Rheum. 2011;63(12):3931-41.
47 Sciascia S, Radin M, Yazdany J, Levy RA, Roccatello D, Dall'Era M, et al. Efficacy of belimumab on renal outcomes in patients with systemic lupus erythematosus: a systematic review. Autoimmun Rev. 2017;16(3):287-93.

48 Chen DY, Chen YM, Wen MC, Hsieh TY, Hung WT, Lan JL. The potential role of Th17 cells and Th17-related cytokines in the pathogenesis of lupus nephritis. Lupus. 2012; 21(13):1385-96.

49 Avihingsanon Y, Phumesin P, Benjachat T, Akkasilpa S, Kittikowit V, Praditpornsilpa K, et al. Measurement of urinary chemokine and growth factor messenger RNAs: a noninvasive monitoring in lupus nephritis. Kidney Int. 2006;69(4):747-53.

50 Abdel Galil SM, Ezzeldin N, El-Boshy ME. The role of serum IL- 17 and IL- 6 as biomarkers of disease activity and predictors of remission in patients with lupus nephritis. Cytokine. 2015 Dec;76(2):280-7.

51 Tucci M, Lombardi L, Richards HB, Dammacco F, Silvestris F. Overexpression of interleukin-12 and Thelper 1 predominance in $\mathrm{lu}$ pus nephritis; 2008.

52 Toward a Liquid Biopsy for Lupus Nephritis: Urine Proteomic Analysis of SLE Identifies Inflammatory and Macrophage Signatures ACR Meeting Abstracts. https://acrabstracts. org/abstract/toward-a-liquid-biopsy-for-lupus-nephritis-urine-proteomic-analysis-ofsle-identifies-inflammatory-and-macrophage-signatures/. Accessed February 4, 2021. 